\title{
Vertebral giant cell tumor: Diagnosis difficulties and therapeutics' challenges
}

\author{
Kherfani Abdelhakim, Amri Khalil, Hachem Mahjoub, Abid Leila, Bouaziz Mouna, \\ Mestiri Mondher
}

Orthopaedic Institute of MT Kassab, Tunisia

Email address:

akhalil.kh@gmail.com (A. Khalil)

To cite this article:

Kherfani Abdelhakim, Amri Khalil, Hachem Mahjoub, Abid Leila, Bouaziz Mouna, Mestiri Mondher. Vertebral Giant Cell Tumor: Diagnosis Difficulties and Therapeutics' Challenges. Journal of Cancer Treatment and Research. Vol. 2, No. 2, 2014, pp. 16-20. doi: $10.11648 /$ j.jctr.20140202.12

\begin{abstract}
Giant cell tumor remains one of the most controversial and discussed bone tumors. Its diagnosis may be very difficult especially in vertebral localization as it requires a careful study of the pathology tissue. The authors report a series of four patients, treated in the MT Kassab orthopaedic Institute for vertebral giant cell tumor (GCT), with different clinical presentation and therapeutic strategies. By presenting those cases we point to surgical and medical treatments' difficulties and the challenge of avoiding local recurrence and distant metastasis of this tumor.
\end{abstract}

Keywords: Giant Cell Tumor, Spine Surgery, Metastasis, MRI, Bisphosphonates

\section{Introduction}

Giant cell tumor (GCT) remains one of the most controversial and discussed bone tumors. Its diagnosis may be difficult and requires a careful imaging and pathological assessment.

En bloc resection when it is possible, is well known to produce the lowest recurrence rate with a higher risk of complications and neurologic deficits [1], intralesional excision can spare nerve roots but must be associated to an adjuvant treatment. However, it has been reported that the treatment options are limited and recurrence rates are higher when GCT arise at a surgical inaccessible location like the spine.

We report a series of four patients, treated in the MT Kassab orthopedic Institute for vertebral giant cell tumor with different clinical presentation and therapeutic strategies.

\subsection{Case 1}

A 48-year-old man with unremarkable medical history presented with a15 days history of subacute thoracic pain. The plain radiographs showed a bilateral hemorrhagic pleurisy, drained by chest tube and a thoracic computed tomography (CT) scan found out a lytic lesion in T10.

(MRI) showed a destructive T10 posterior arch lesion, extensive to soft tissues and posterior to the spinal cord in $\mathrm{T} 10$ with decreased signal intensity on T1-weighted images and increased signal intensity on T2-weighted images and after Gadolinium administration (fig 1).

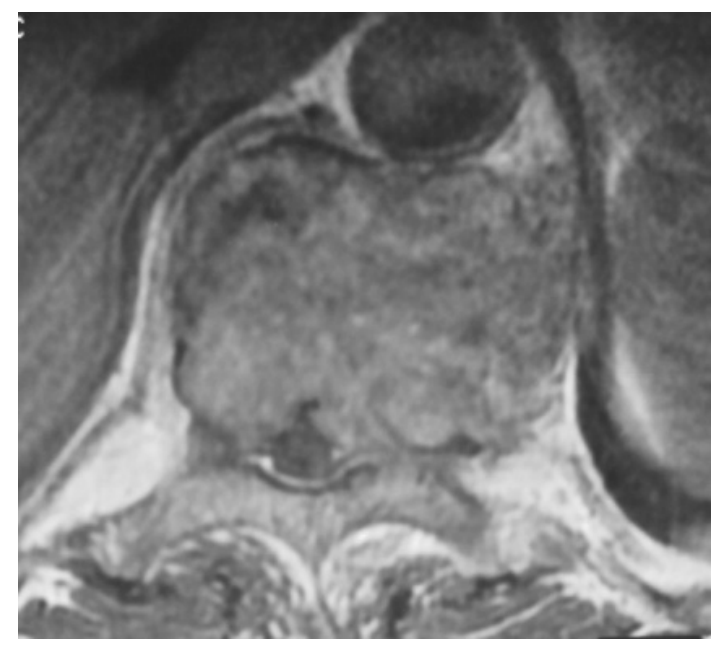

Figure 1. Transversal MRI view showing an expansive lesion of T10 with intermediate signal intensity on T1-weighted images after Intravenous Gadolinium administration

Facing an isolated lytic lesion of the thoracic spine a CT guided biopsy was performed. Microscopic examination showed tumor tissue consisting of thin septa separating 
spaces that containing blood. The septa lack an endothelial lining and consist of oval cells and giant cells. The diagnosis then was an aneurysmal bone cyst (ABC).

We conducted a laminectomy of T10 with bilateral screw fixation of T8-T12 without autologous bone graft associated to a new biopsy of tumoral tissue, first one to confirm the diagnosis of giant-cell-tumor. Indeed, the tumor was composed mainly of mononuclear cells intimately admixed with many large multinucleated giant cells and aneurysmal bone cyst-like areas. Mitotic figures were not readily seen, and necrosis was not present (fig 2). The definitive diagnosis was a GCT with a secondary aneurysmal bone cyst.

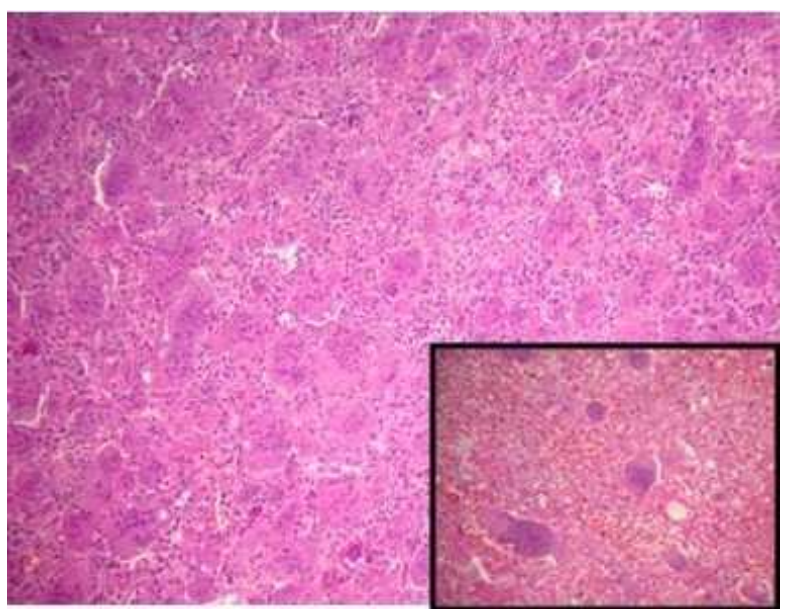

Figure 2. Characteristic mononuclear cells with a large number of giant cells, the multinucloid giant cells had a relatively uniform size and number of nuclei per cell $>10$

Three months later, the patient developed a neurological deficit and a new MRI showed a double sized tumor with intraspinal extension associated to pleural and parenchymal abnormalities (fig 3).



Figure 3. Transversal T2 weighted MRI view showing an increase in the tumor size with left paravertebral mass.

Facing the urgent neurological deficit, we re-conducted the posterior approach for a spinal release, the spinal cord was sheathed by the tumor and the resection was subtotal.
Despite this the patient presented a significant improvement of his neurological status.

The patient underwent the antero-lateral approach, but nor corporectomy or stabilization was performed due to hemorrhage. Histologically, there was no malignant transformation of the GCT. Because of the tumor size, the surrounding soft tissues involvement and the non radical tumor excision, our patient presents a high risk of recurrence, and a postoperative radiotherapy was conducted with a total dose of 45 Gy associated to adjuvant therapies with aminobiphosphonate. The patient has since a steady neurological status, and a new MRI, three month later, showed a partial regression of tumor volum.

\subsection{Case 2}

A 28-year-old woman presented for mid-back pain with no prior symptoms. Plain radiographs, CT and MRI, showed an expansile osteolytic lesion of L1 body extending to the right posterior elements (fig 4).

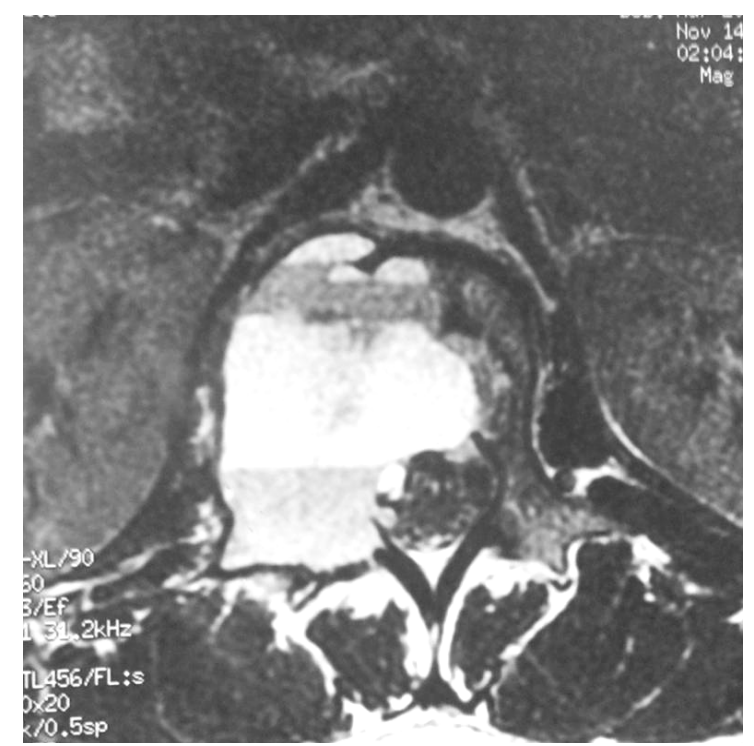

Figure 4. Transversal T2 weighted MRI view: Expansile lesion of L1 body extending to the right posterior elements with fluid-fluid levels

A CT guided biopsy was performed. On pathological examination, the tumor consists in vascular channels lined by osteoclastic giant cell associated to areas of hemorrhage. The diagnosis was an ABC.

Two weeks later the patient presented a L1 pathological fracture secondary to the extension of the tumor volum with no neurological complications.

Facing this unusual evolution and the fast extension of the tumor, surgical treatment with a second intraoperative biopsy was indicated. The treatment consisted in intralesional excision through a combined anterior and posterior approach with extended vertebral fixation and autologous bone graft, made after a preoperative embolization. The new pathology tissue was examinated. It was composed of spindle cells and multinucleated giant cells. Areas with a fascicular pattern devoid of giant cells, 
prominent cystic vascular spaces and large areas of hemorrhage were noted. A definitive diagnosis of GCT was made with area of secondary $\mathrm{ABC}$.

The patient fully recovered after the surgery and was able to continue her regular daily activities.

One year later, she progressively developed worsening back pain. A CT scan was performed, showing a large soft tissue mass postero-lateral to the spine and arising from the previously operated area with a fortuitous discovery of pulmonary multiple nodules (fig 5).

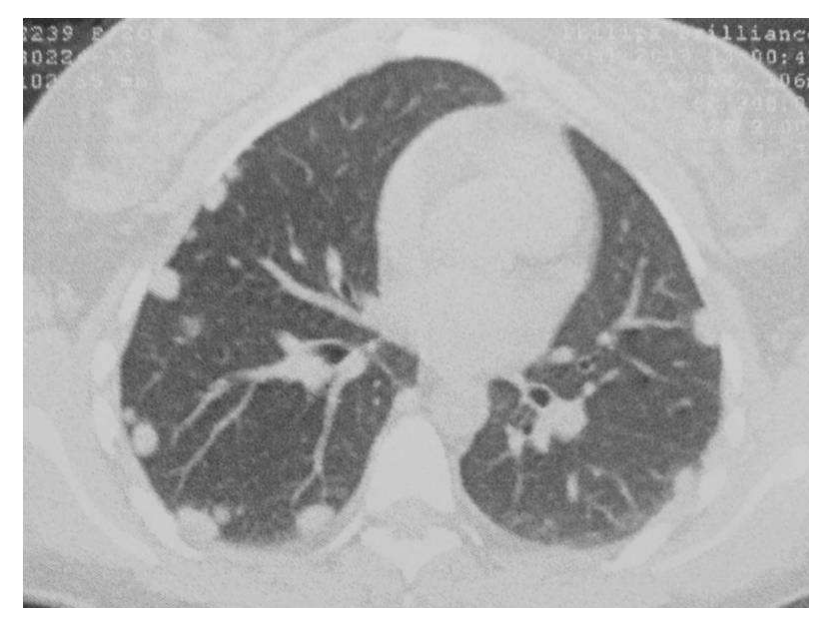

Figure 5. Transversal CT view in pulmonary algorithm: Multiple pulmonary nodules

The patient had a second intralesional excision through a posterior approach associated to a metastectomy of multiple lung nodules. On macroscopy, the lung metastatectomie had a well-circumscribed nodular appearance. The histological features are identical to the vertebral lesion however peripheral areas of reactive bone formation were seen. The diagnosis of lung metastases of GCT was confirmed (fig 6).

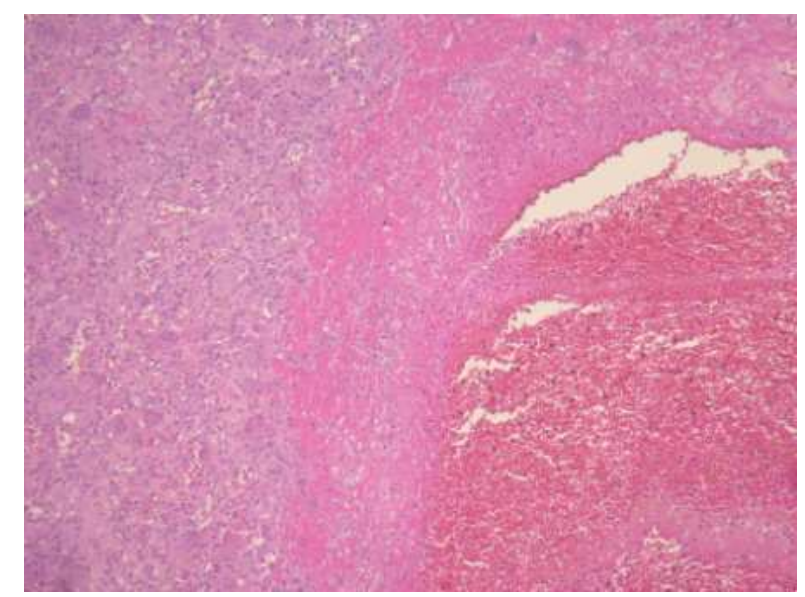

Figure 6. Pulmonary metastasis with typical microscopic appearance of a giant cell tumor with reactive bone formation at the periphery of the tumor nodule

An adjuvant therapy with aminobiphosphonate was performed associated to radiation for the local lesion. At the latest follow-up (thirteen months), two $\mathrm{CT}$ scan made at three and six months after the second surgery, showed no growth of the tumor volume.

\subsection{Case 3}

A 27 years old woman, presented for an acute monoplegia of the right lower limb and urinary incontinence. Physical examination showed marked pyramidal signs in the right lower limb.

CT scan and MRI showed a suspicious lesion of L1 (fig 7), with an extension to soft tissue.



Figure 7. Transversal CT view I bone algorithm: expansile osteolytic lesion of L1 posterior elements with extension to vertebral body, adjacent rib and soft tissues

Facing this neurological emergency, surgery was conducted, and the patient had an en bloc resection of the mass associated to an extended posterior fixation, with a biopsy interpreted as GCT.

At the last follow up of two years, the patient showed no local recurrence or distant metastases.

\subsection{Case 4}

A 47 year-old man, his first complaint was a spontaneous lumbar radicular pain in the left L3 territory. The neurological examination showed a minor deficit on the left L3 and L4 roots, coated 4 in muscle testing.

Standard AP and lateral radiography coupled with myelography objectified a purely lytic bone defect with fuzzy boundaries associated with a posterolateral compaction of the body of L3, realizing the aspect of blind vertebra. Incomplete interruption of the opaque column, extradural type, in front of L3.

The myelo-CT axial section with sagittal reconstruction confirmed the partitioned geographic osteolysis with tissular density of the L3 hemi vertebra, associated with a left anterolateral epiduritis compressing and repressing the dural sac.

Initially, the patient underwent a decompressive laminectomy and an L1-L4 fixation, with transpedicular biopsy of the tumor. The diagnosis of CGT has been established (fig 8). 




Figure 8. (Middle power $x$ 200) GCT with area of Secondary ABC

A second operation through an anterior approach consisted of a tumoral recess with curettage, aspiration and L2-L3, L3-L4 discectomy.

Macroscopically, the tumor excision was judged complete. An inter-body bone graft was performed. The postoperative course was uneventful and the evolution was marked by the complete disappearance of the lumbar and radicular pain. Patient follow-up was quarterly the first year, biannual during the second and third year.

At five years after surgery, the patient consulted again for weakness of both lower limbs, gradually developed, limiting the walk perimeter to $500 \mathrm{~m}$. Neurological examination objectified pure motor para-paresis grade 4 . Radiographs showed lysis around the fibular graft with segmental lumbar kyphosis and collapses of the body of L5. The MRI revealed a highly evocative appearance of tumor recurrence. A CT guided biopsy there was no malignant transformation of the GCT.

\section{Discussion}

GCT of the spine is found in less than $7 \%$ of all bone GCT, in which the thoracic spine is rarely involved. The biological behavior of GCT ranges from latent, active to locally aggressive forms and occasionally distant metastasis [2].Radiographs and CT usually show an expansile osteolytic lesion usually arising from vertebral body and extending to the adjacent soft tissues. Fluid-fluid level may be observed $n$ some cases; usually related to associate ABC. MRI allows a precise locoregional assessment especially to the spinal canal. Histopathology confirms the diagnostic with the presence of numerous rounds, oval or spindleshaped mononuclear cells and a very large numbers of osteoclast-like giant cells scattered uniformly throughout the tumor.

Histological differentiation of primary GCT of bone from other giant cell containing tumors is problematic and made more challenging as aneurysmal bone cysts and giant cell tumors of bone may co-exist [3]. That was the difficulty observed in the first two cases, the differential diagnosis with an aneurysmal bone cyst is a problem especially in cases of needle core biopsies.
In the literature, there are reported cases of GCT in the thoracic spine with different treatments and outcome [4].

Surgical treatment of a thoracic vertebra GCT needs to be individualized depending on the local extension, the contact with soft tissue and the type of presentation. Marginal en bloc resection is possible only if the GCT is limited to vertebral body, but if the tumor invades posterior elements then the corpectomy will not be sufficient [5] and should be associated to preoperative embolization to control hemorrhage and prevent recurrences. In our experience, recurrence can be observed even if resection is judged complete on the macroscopic level.

When there is a recurrence of GCT, with or without metastases, the local recurrence and possibly the metastases must be biopsied to confirm the original diagnosis. The behavior of the lung lesions, observed in $2 \%$ of bone GCT [6], is somewhat unpredictable, with a small percentage regressing with or without any adjuvant treatment [7]. In the second case, the appearance of lung metastasis within such a short period might be explained by surgical manipulation. Indeed, surgical manipulation and embolization have been associated with an increased risk of metastasis. In point of fact, it is currently hypothesized that metastases result from emboli that travel to distant sites from the original tumor [8].

In our cases, we point out the fact that local extension of the tumor may be unpredictable, especially if the tumor tends to spread even in non bony structures such as lung parenchyma, or posterior to the spinal cord, causing spinal cord compression. This last evolution leaves no option but surgery sometimes in non optimal conditions.

Surgical treatment is advised but not always sufficient due to the anatomic complexity of the spine, the presence of important blood vessels, the intimate contact with the dura mater or the pleura. In such cases, adjuvant therapies with an aminobiphosphonate or more specifically the antiRANKL have been shown to delay or stop tumor growth [9].

Radiotherapy for giant cell tumors of bone is discussed as postoperative treatment in cases of non radical tumor excision, or as a palliative treatment of postoperative recurrences or for unresectable large tumors [10]. Tumor size and the involvement of surrounding soft tissues have been found to be two major prognostic factors [11].

\section{Conclusion}

Optimal management of GCT of bone is complete resection of the tumor with wide margins if possible. In case of thoracic-spine localization with local extension, en bloc resection seems to be very difficult and preoperative embolization must be discussed before surgery. For large tumors with high risk of soft tissue involvement, radical surgery, when possible, should be considered as primary treatment combined with adjuvant therapies with an aminobiphosphonate. The behavior of the lung lesions is somewhat unpredictable, with a small percentage 
regressing with or without any adjuvant treatment.

\section{References}

[1] Liu HS, Wang JW. Treatment of giant cell tumor of bone: A comparison of local curettage and wide resection. Chang Keng I Hsueh 1998; 21:37- 43.

[2] Fletcher CDM, Bridge JA, Hogendoorn PCW, Mertens F (eds) - WHO Classification of tumours of soft tissue and bone. IARC: Lyon 2013.

[3] Su YP, Chen WM, Chen TH. Giant cell tumors of bone: an analysis of 87 cases. Int Orthop 2004;28:239-43.

[4] Oda Y, Miura H, Tsuneyoshi M, et al. Giant cell tumor of bone: Oncological and functional results of long-term follow-up. Jpn J Clin Oncol 1998; 28:323-328.

[5] Sanjay BK, Sim FH, Unni KK, McLeod RA, Klassen RA. Giant-cell tumours of the spine. J Bone Joint Surg Br. 1993; 75:148-154. [PubMed]
[6] S.Jacopin and al. Fatal lung metastasis secondary to index finger giant cell tumor in an 8-year-old child. Orthopaedic and Traumatology: Surgery and Research (2010) 96, 310313.

[7] Cheng JC, Johnston JO (1997) Giant cell tumor of bone. Prognosis and treatment of pulmonary metastases. Clin Orthop Relat Res 338:205-214

[8] Jacopin and al. Fatal lung metastasis secondary to index finger giant cell tumor in 8-year-old child. Orthopaedics and traumatology: surgery and research (2010) 96, 310-313

[9] Lung Fung Tse and al. Bisphosphonates reduce local recurrence in extremity giant cell tumor of bone: A casecontrol study. Bone 42 (2008) 68-73

[10] Khan DC, Malhotra S, Stevens RE, et al. Radiotherapy for the treatment of giant cell tumor of the spine: a report of six cases and review of the literature. Cancer Invest 1999; $17: 110-113$.

[11] Masui F, Ushigome S, Fujii K. Giant cell tumor of bone: A clinicopathologic study of prognostic factors. Pathol Int 1998; 48:723-729. 